\title{
Applications of Multi-object Spectroscopy - A Large Sample of Carbon Stars in the LMC
}

\author{
Russell Cannon and Alison Offer \\ Anglo-Australian Observatory, PO Box 296, Epping, NSW 2121, \\ Australia
}

Despina Hatzidimitriou and Barry Croke

Physics Department, University of Crete, PO Box 2208, GR 710 03, Heraklion, Crete, Greece

David Morgan

Royal Observatory, Blackford Hill, Edinburgh EH9 3HJ, Scotland, UK

\begin{abstract}
The application of multi-object instruments to nearby galaxies permits the spectroscopy of large samples of faint stars. Such data are needed to determine the kinematics and metallicity distributions of stellar systems, to complement the excellent photometric data now becoming available. Together, these data should reveal the star formation and evolutionary histories of Local Group galaxies. The Two-degree Field (2dF) instrument on the Anglo-Australian Telescope is described and some initial results are presented for a sample of 700 carbon stars in the Large Magellanic Cloud. The velocity precision is more than adequate for kinematic studies, while the application of Principal Component Analysis techniques promises to yield an objective and quantitative classification scheme for carbon stars. This may lead to a better understanding of the carbon stars themselves, as well as enhancing their use as tracers of the intermediate-age and old stellar populations. Another application of multi-fibre spectroscopy, to a sample of main sequence stars in the globular cluster 47 Tuc, illustrates the possibility of combining the data for many faint stars to yield composite spectra with relatively high signalto-noise ratio.
\end{abstract}

\section{The Case for Multi-object Spectroscopy}

One of the surprises that arose during the preparations for IAU Symposium 192 was the imbalance between photometric and spectroscopic data. By analogy with recent developments in observational cosmology, where the combination of HST imaging and Keck spectroscopy is producing tremendous advances in the study of high redshift galaxies and quasars, we had expected that the most powerful facilities would already be in widespread use for the spectroscopy of stars in Local Group galaxies. However, the contributions to this meeting indicate that this has barely begun to happen. 
The requirements of stellar spectroscopy are much more stringent than those for determining the redshifts of distant emission-line galaxies. To do even crude abundance work requires spectra with typically $2 \AA$ resolution, i.e. a resolving power of a few thousand, and Signal-to-Noise ratio $(\mathrm{S} / \mathrm{N})$ of a few tens, to permit measurement of the stronger stellar absorption features. Accurate abundance measurements require much higher resolution and S/N. Kinematic studies similarly require at least moderate resolution, in systems with ranges in velocity of only a few tens of $\mathrm{km} \mathrm{s}^{-1}$. High spatial resolution is also generally needed, to obtain uncontaminated spectra in crowded star fields. These criteria make it difficult for spectroscopy to match our imaging capabilities; even a moderately small ground-based telescope can readily produce excellent CMDs for stars which are much too faint for useful spectroscopy. Clearly it is not feasible to obtain spectra for significant numbers of stars if each one requires an exposure time of hours.

The solution to this problem is multi-object spectroscopy, using either optical fibres or multi-slits. This can help in two ways: by providing large samples of individual stars in a few nights of observing time, or by enabling spectroscopy to much fainter limits, through using long exposures and also combining spectra for many stars. Here we describe examples of both types, based on work with the fibre systems on the Anglo-Australian Telescope (AAT).

\section{The Two-degree Field (2dF) Instrument on the AAT}

The $2 \mathrm{dF}$ instrument is the latest in a line of multi-object fibre systems built for the AAT. It has two main advantages over the earlier machines: as its name implies, it covers a field of view which is two degrees in diameter at the prime focus; and it can observe 400 objects simultaneously. Thus in terms of both sky coverage and numbers of objects it is almost an order of magnitude more powerful than its predecessors, the FOCAP (Gray, 1986) and Autofib (Parry \& Sharples, 1988) instruments at the Cassegrain focus. In addition, it is equipped with a pair of custom-built spectrographs that are at least twice as efficient as the old RGO Cassegrain spectrograph, and it has fibres and CCD detectors with higher throughput, especially in the ultraviolet.

The $2 \mathrm{dF}$ instrument has been described a number of times (e.g. Lewis, Glazebrook \& Taylor, 1998; Smith \& Lankshear, 1998) so only a brief outline is given here. All of the components of $2 \mathrm{dF}$ are mounted on a new demountable top-end ring for the AAT. The corrector lens is a four-element design which incorporates an atmospheric dispersion compensator. The fibres are mounted in retractors around the edges of circular steel field plates. Each fibre terminates in a small metal block incorporating a permanent magnet in its base; a tiny 45 degree prism is cemented on to the end of each fibre. A robot positioner is used to pick and place each of the 400 fibres sequentially. Since this is a time-consuming process there are two field plates and two complete sets of fibres, which can be interchanged by a tumbler mechanism. Thus the robot can configure one set-up while another set of targets is being observed. The configuration time is about one hour, which is well-matched to the typical exposure times for many projects, while the time to switch fields and acquire a new set of targets is only about five minutes. 
All of the fibres are led from the prime focus down to a pair of spectrographs mounted on the periphery of the new top-end ring. This keeps the fibre runs short, to maximise the UV throughput. It also means that there is no need to disconnect and reconnect fibres when $2 \mathrm{dF}$ is put on or off the telescope; the whole instrument is handled as a unit. Two spectrographs were used simply because the largest readily available detectors when $2 \mathrm{dF}$ was being designed had formats of about 1000-square pixels. 200 fibres are led to each spectrograph (actually, two interchangeable sets of 200 to each, corresponding to the two field plates), so that there are five rows of pixels available for each spectrum. Most of the signal is in two or three pixels, so that the spectra can be readily separated and extracted; the light scattered into adjacent spectra is usually less than one percent. Acquisition and guiding of the fields is done by a combination of an imaging TV system and a set of special fibre bundles, each of which is located on a relatively bright reference star.

All of 2dF's functions are under computer control, using a windows system which enables the observer to drive the telescope, the spectrographs, the datataking and the fibre positioner from a single console. Data reductions are also highly automated; the $\mathbf{2 d f d r}$ system performs a sequence of operations involving spectrum extraction, wavelength calibration, sky subtraction and frame combination. The entire procedure can be run automatically and the results of an observation are available within ten minutes of the end of a series of exposures, in the form a set of fully calibrated spectra, complete with diagnostic statistics on the efficiency with which targets have been hit and the $\mathrm{S} / \mathrm{N}$ of the data.

\section{A Large Sample of Carbon Stars in the Large Magellanic Cloud}

The Magellanic Clouds are prime targets for multi-object spectroscopy. One early application was the use of the AAT's Autofib system to look at the kinematics of stars in the red giant clump in the SMC (Hatzidimitriou, Cannon \& Hawkins, 1993). Previous photometric studies, based on very large samples of stars measured on UK Schmidt Telescope photographs, had shown that while the clump was well-defined over most of the face of the SMC, it extended over a larger magnitude range in the outer NE quadrant. Was this a consequence of differential reddening, did it represent a greater range of intrinsic luminosities for some reason, or did it mean that the SMC is more extended along that line of sight?

Spectra for a sample of about 50 SMC clump stars with $18.2<R<19.2$ were obtained over two nights, with a total exposure time of over 4 hours. 31 of the stars had spectra with $\mathrm{S} / \mathrm{N} \sim 5-10$, sufficient to yield velocities accurate to $\pm 10 \mathrm{~km} \mathrm{~s}^{-1}$. The velocities showed a clear correlation with apparent magnitude, with the fainter stars receding more rapidly. The simplest interpretation is that the NE side of the SMC is being stretched out along the line of sight. The observed rate of $8 \mathrm{~km} \mathrm{~s}^{-1} \mathrm{kpc}^{-1}$ implies a timescale of $\sim 1.2 \times 10^{8} \mathrm{yr}$, consistent with this being an effect of a recent close encounter with the LMC.

While clump stars certainly trace the intermediate-age and old stellar populations, it is hard to be certain about the status or intrinisic luminosity of individual stars. Carbon stars provide a better tracer of this population, since they can be unambiguously identified from Schmidt objective prism photographs 


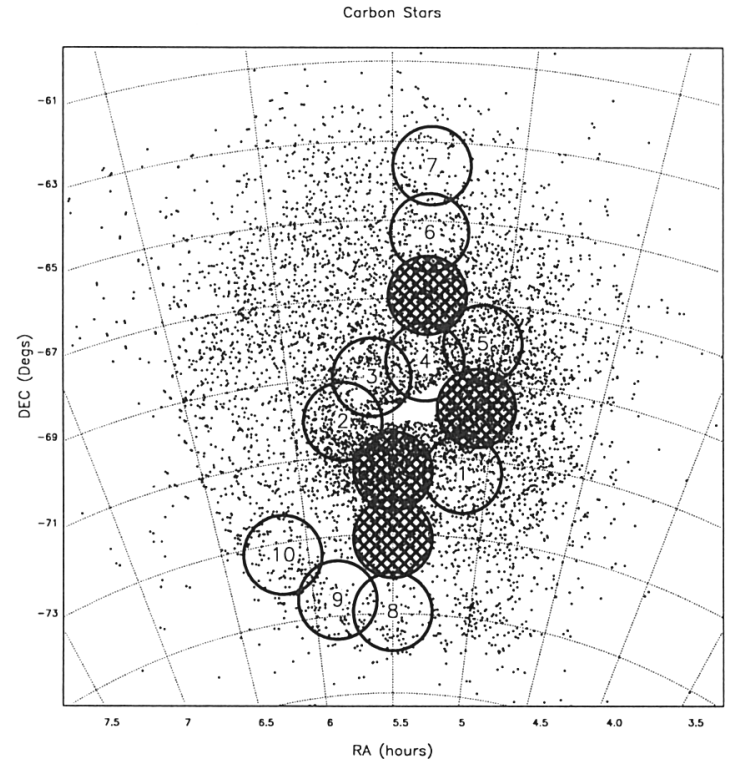

Figure 1. The distribution of carbon stars in the LMC, and the planned location of $2 \mathrm{dF}$ fields. The cross-hatched circles are the four fields observed in January 1998.

and it is easy to define samples with very high completeness and reliability. Two other advantages of the carbon stars are that they are about an order of magnitude brighter than the clump, and they have many sharp features making it easy to determine accurate radial velocities. A programme is underway to obtain spectra for large samples of carbon stars in both Clouds, using $2 \mathrm{dF}$.

\subsection{The 2dF Magellanic Clouds carbon star programme}

The principal aim of the programme is to derive large scale maps of the mean velocities and velocity dispersions across the full extent of both Clouds. These will be compared with similar data for younger stellar populations, and for the interstellar gas, to try to understand the kinematic and dynamic histories of the Clouds. One specific objective is to try to distinguish between the internal motions and the pattern speed of the LMC bar, following the method of Tremaine \& Weinberg (1984). Another aim is to use the data to better understand the carbon stars themselves: by combining kinematic, photometric and spectroscopic data, it may be possible to identify different populations of carbon stars, corresponding to progenitors of different metallicities and masses, and hence ages.

Target stars have been selected primarily from the extensive lists of carbon stars found on UKST prism plates (Morgan \& Hatzidimitriou, 1995 and Dapergolas et al., 1997). For the main LMC project, a series of slightly overlapping $2 \mathrm{dF}$ fields has been selected, lying along a N-S axis which corresponds approximately to the line of nodes (Fig. 1). Together they cover a strip about $13^{\circ}$ long; stars in the overlaps will be used to ensure a consistent velocity system for the 

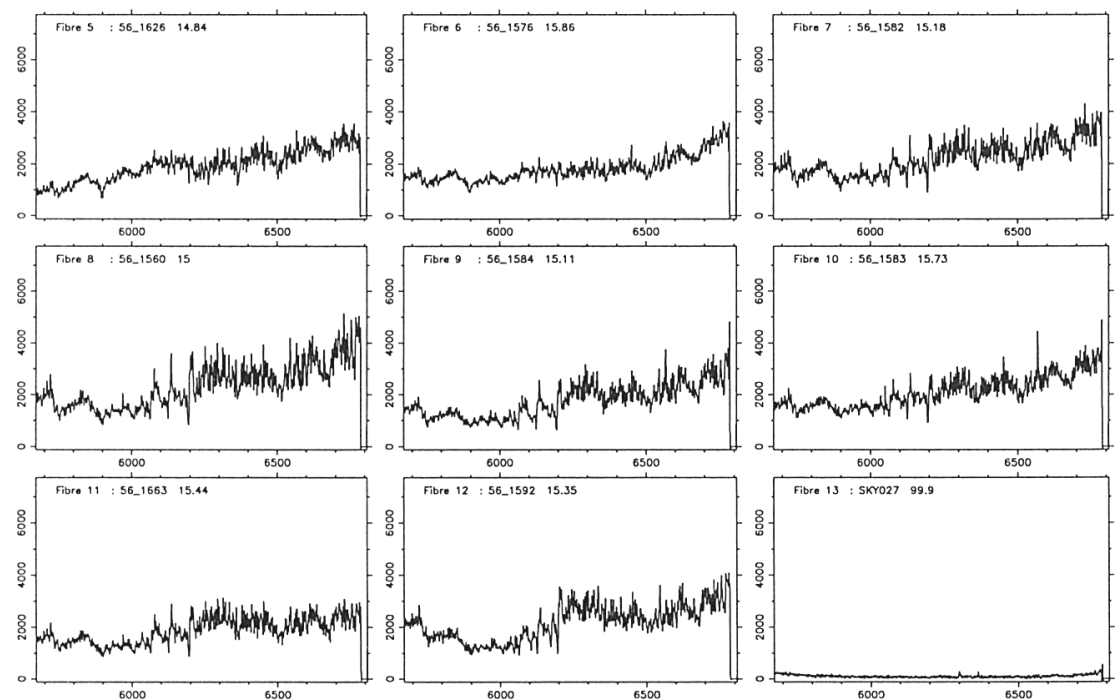

Figure 2. Typical 2dF C star spectra. The central panel shows a common sub-type, with strong $\mathrm{C}_{2}$ bands; the middle panel in the bottom row shows a 'J-type' star, with doubling of the bands due to enhanced isotopic ${ }^{13} \mathrm{C}$ abundance.

whole set. The number of targets per $2 \mathrm{dF}$ field ranges from about 350 stars in the centre (about two dozen of the 400 fibres are placed on clear sky positions, and currently about two dozen fibres are out of use) to fewer than 100 in the outermost fields, where the yield is limited by the low surface density of carbon stars. The samples in some of the outer fields have been augmented by including stars selected on the basis of DENIS infrared photometry (see paper by Cioni et al. at this meeting). A smaller sample of SMC carbon stars is also included, principally for comparison with the LMC population.

\subsection{Results to date}

Approximately $700 \mathrm{LMC}$ carbon stars in four $2 \mathrm{dF}$ fields were observed during two partially clear nights in January 1998. The spectra covered a range of $1100 \AA$ centred at $6250 \AA$, to include several $\mathrm{C}_{2}$ and $\mathrm{CN}$ bands as well as lines of $\mathrm{Na}$, $\mathrm{Ba}$ and $\mathrm{Li}$. Exposure times of 40-45 min yielded spectra with $\mathrm{S} / \mathrm{N}$ about 50 per pixel for stars with $R \sim 15$; this is considerably higher than necessary for determining radial velocities and means that the spectra are suitable for detailed classification work and abundance analyses. There was no point in curtailing the exposures since the total time per field, including arcs and sky exposures, was about 75 minutes, which is the same as the time currently needed for the $2 \mathrm{dF}$ robot to reconfigure a full set of 400 fibres. Some representative spectra are shown in Fig. 2.

The spectra were cross-correlated with a synthetic spectrum and with a standard star spectrum, to yield radial velocities with an internal accuracy of about $2.7 \mathrm{~km} \mathrm{~s}^{-1}$, better than expected for a resolution of $1.1 \AA$ pixel $^{-1}$. Little 

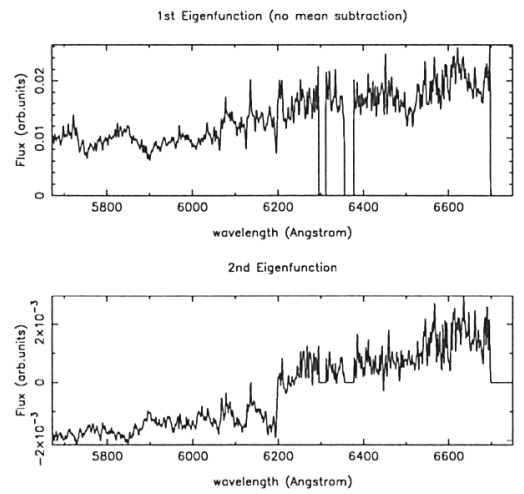

3rd Eigenfunction

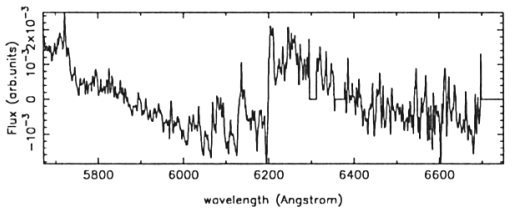

Figure 3. The first three eigenspectra from a PCA analysis of a subset of the LMC C star spectra.

analysis has been done so far on this initial sample, but the velocity dispersion is about $25 \mathrm{~km} \mathrm{~s}^{-1}$ and there is a N-S trend in the mean velocity which is consistent with the mean rotation determined by Kunkel et al. (1997).

\subsection{Automatic spectral classification}

Having such a large sample of spectra makes it feasible to use automatic classification techniques; indeed it makes it almost essential to do so. One such technique is Principal Component Analysis (PCA), which looks at all available spectra in the $\sim 1000$-dimensional spectral wavelength space and finds the principal components or eigenspectra (Glazebrook, Offer \& Deeley, 1998). This has been applied so far to one set of about $1502 \mathrm{dF}$ spectra, with the results shown in Fig. 3 .

The first eigenspectrum, effectively the mean spectrum of the sample, is very similar to the 'typical' spectra in Fig. 2, e.g. in fibres 7 and 10; the second eigenspectrum has strong $\mathrm{C}_{2}$ bands similar to fibre 9 , while the third shows the isotopic doubling of the bands seen in fibre 12 in Fig. 2.

The relative strengths of the eigenspectrum components give a quantitative description of each spectrum. The validity of the PCA classification can be checked by looking at the correlation of the strengths of the eigenspectra with observationally independent parameters; for example, in an $R$ versus $(R-I)$ CMD, the stars with a strong component of the third eigenspectrum, the J-type stars, are generally well separated from stars with a weak third component.

This preliminary result is very encouraging. It not only suggests that a good automatic classification of the carbon stars should be possible, one which is both objective and quantitative, but that this can be readily tied to more traditional 


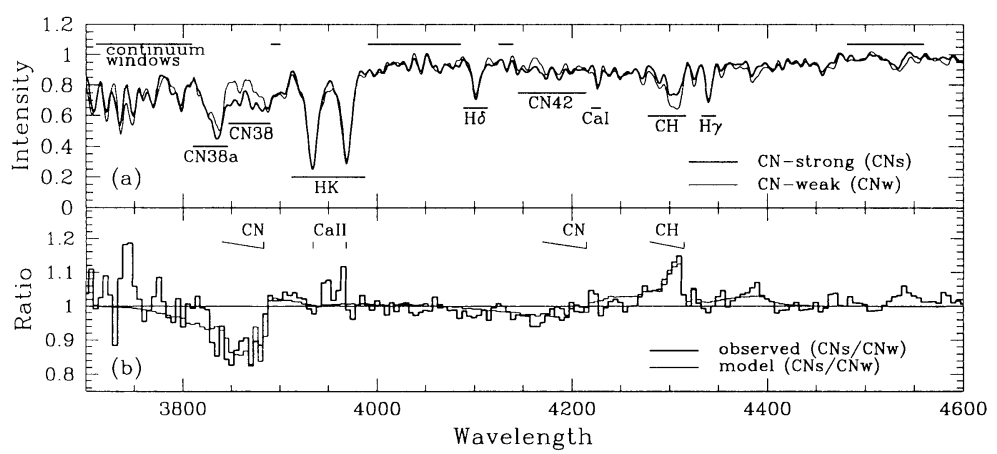

Figure 4. Very long exposure (40 hours) composite spectra of main sequence stars in 47 Tuc. The upper panel compares the observed spectra for groups of $\mathrm{CN}$-strong and weak stars; the lower panel gives the ratio of these two spectra, compared with the same ratio for a pair of synthetic spectra with appropriate abundances.

classification schemes. This in turn should make it possible to interpret the classification in terms of physically meaningful parameters, and to investigate how these parameters correlate with other properties of the stars such as their velocities and magnitudes. The ultimate hope is that this will yield a way of dividing the $\mathrm{C}$ stars into sub-populations of different ages and origins, which in turn would feed back into the kinematic analysis of the LMC.

\section{Application to Faint Main Sequence Stars in Globular Clusters}

The carbon star project described above is an application of $2 \mathrm{dF}$ where the crucial factor is the ability to observe very large numbers of stars, spread over a substantial area of sky. A somewhat different situation arises in the spectroscopy of fainter stars, such as main sequence stars in Galactic globular clusters. There the key factor is the ability to observe the same stars for many hours, while observing a large enough sample simultaneously to keep the total telescope time down to a few nights. Even larger gains can be made in special circumstances, if the spectra of many stars can be combined. An example of both techniques is given by some old but only recently analysed AAT data for 47 Tucanae.

In this case, FOCAP was used to observe a hundred faint stars $(17.0<$ $V<18.6$ ) for several hours each, to look for variations in the strengths of the ultraviolet $\mathrm{CN}$ and blue $\mathrm{CH}$ bands. The spectra were too noisy to unambiguously classify the individual stars. However, it proved possible to subdivide the sample by using synthetic band strength indices. Having split the stars into 'CN-strong' and 'CN-weak' groups, it was easy to show that the strength of the CN feature was anti-correlated with $\mathrm{CH}$. Furthermore, by combining the spectra of 8 strong and 8 weak stars, two composite spectra with effective total exposure times of 40 hours were created (Fig. 4, upper panel).

These composite spectra have sufficient $\mathrm{S} / \mathrm{N}$ to carry out an abundance analysis by comparing the observed spectra with a grid of synthetic model spectra. 
This showed that the variations in the main sequence stars could be explained by the same range of $\mathrm{C}$ and $\mathrm{N}$ abundances as is known to occur in evolved red giants, showing that the variations in 47 Tuc are probably primordial in some sense, and cannot be due simply to dredge-up of processed material from within the stars themselves. The validity of the initial separation of the stars, on the basis of the UV CN band, was confirmed by the emergence of a much weaker blue $\mathrm{CN}$ feature and by the detailed structure of the $\mathrm{CH}$ band in the composite spectra (see the lower panel of Fig. 4). Full details of this work have been published (Cannon et al. 1998).

\section{Prospects for the Future}

Perhaps the biggest problem with a major new facility like $2 \mathrm{dF}$ is being selective, and deciding which projects are the most important ones to pursue. Clearly there is a way of obtaining intermediate dispersion spectroscopy for large samples of stars down to about magnitude 19, although the maximum resolution attainable with $2 \mathrm{dF}$, using 1200 line $\mathrm{mm}^{-1}$ gratings in first order, is not as high as one would like for some purposes, such as separating stellar and interstellar components of absorption lines or detecting binarity through radial velocity shifts. A doubling of the available resolution should be possible by working in second order, or perhaps using holographic transmission gratings, but these options have not so far been implemented in $2 \mathrm{dF}$.

However, two problems are not addressed by $2 \mathrm{dF}$ or systems like it on $4 \mathrm{~m}$ telescopes. One is to get reasonably high $\mathrm{S} / \mathrm{N}$ spectra for yet fainter stars, to learn more about the stars for which we now have excellent CMD data but no spectroscopic information, such as old main sequence stars in the Magellanic Clouds, turn-off stars in dSph galaxies and giants in M31 and its companions. This problem should be addressed when multi-slit or fibre systems become available on $8 \mathrm{~m}$ class telescopes, with their twin advantages of larger aperture and higher spatial resolution.

The second problem is to get good enough spectra for full abundance analyses of individual stars; this requires higher resolutions of about 20000 , combined with high S/N. A start has been made by using echelle spectrographs on $4 \mathrm{~m}$ telescopes, looking at the brightest evolved stars in the Magellanic Clouds and Galactic globular clusters. Similar spectrographs on the Keck and other new telescopes are beginning to be used for some stellar work and give substantial gains, but the exposure times are still prohibitive for most projects. Of currently available multi-object systems, only Hydra at KPNO can work at high enough dispersion. What is needed is a multiplexing system yielding high dispersion on a very large telescope, able either to observe many stars in a single order, or to interlace multi-order echelle spectra for several stars. Until this problem is solved the detailed spectroscopic study of stars in the Local Group will continue to be very hard work.

Acknowledgements. None of the $2 \mathrm{dF}$ data described above, or indeed of the earlier FOCAP and Autofib data, could have been obtained without the involvement and assistance of a very large number of people, on the staff of the AAO and elsewhere. We thank all of the people who have contributed to creating and operating these powerful facilities. 


\section{References}

Cannon, R.D., Croke, B.F.W., Bell, R.A., Hesser, J.E., Stathakis, R.A. 1998, MNRAS, 298, 601

Dapergolas, A., Kontizas, E., Morgan D.H., Kontizas, M. 1997, in: Wide Field Spectroscopy, (eds.) E. Kontizas et al., Astrophys. Sp. Sci. Lib., 212, 161

Glazebrook, K., Offer, A.R. Deeley, K. 1998, ApJ, 492, 98

Gray, P.M. 1986, SPIE Proc., 627, 96

Hatzidimitriou, D., Cannon, R.D., Hawkins, M.R.S. 1993, MNRAS, 261, 873

Kunkel, W.E., Demers, S., Irwin, M.J., Albert, L. 1997, ApJ, 488, L129

Lewis, I.J., Glazebrook, K., Taylor, K. 1998, SPIE Proc., 3355, 828

Morgan, D.H., Hatzidimitriou, D. 1995, A\&AS, 113, 539

Parry, I.R., Sharples, R.M. 1988, in: Fibre Optics in Astronomy, (ed.) S.C. Barden, ASP Conf. Ser., 3, p. 93

Smith, G., Lankshear, A. 1998, SPIE Proc., 3355, 905

Tremaine, S., Weinberg, M.D. 1984, ApJ, 282, L5

\section{Discussion}

Filipovic: Very nice results regarding the depth in the SMC. What about depth in the LMC? Shall we expect significant depth in the LMC?

Cannon: I'm sure there will be structure if we look closely enough. We picked out the NE section of the SMC first because the elongation along the line of sight seemed greatest there.

O'Donoghue: Is the reason to put the spectrographs on the top end of the $2 \mathrm{dF}$ to avoid light losses in the fibres?

Cannon: Yes, especially at the ultraviolet end of the spectrum. And to avoid problems in connecting and disconnecting hundreds of fibres. But we sacrifice something in spectroscopic stability.

Bersier: How did you determine your accuracy in velocity? Is it internal or external?

Cannon: Both. We had some stars in common in two overlapping 2dF fields, for at least a semi-external error. We could also get the internal errors by comparing separate exposures of the same field. Both gave the same result, of just over $2.5 \mathrm{~km} \mathrm{~s}^{-1}$. 